\title{
Desvenlafaxine-Induced Interstitial Pneumonitis: A Case Report
}

\author{
Arjan Flora $^{1}$ (D) Daniel Pipoly ${ }^{2}$
}

Published online: 17 January 2018

(c) The Author(s) 2018. This article is an open access publication

\begin{abstract}
A 52-year-old man developed interstitial pneumonitis during treatment with desvenlafaxine for major depressive disorder. The man received desvenlafaxine at $50 \mathrm{mg}$ for symptoms of depression 4 years earlier. Six months after a dose increase to $100 \mathrm{mg}$, he developed bronchitic symptoms with mild, persistent dyspnea. Investigations revealed a restrictive pattern on pulmonary function testing, bilateral upper lobe reticular opacities with traction bronchiectasis on radiology imaging, and endstage interstitial fibrosis with honeycomb changes consistent with chronic hypersensitivity pneumonitis on open lung biopsy. He was diagnosed with drug-induced interstitial pneumonitis. Desvenlafaxine was discontinued and the patient received prednisone and mycophenolate mofetil. The patient had subsequent stability in the progression of his pulmonary disease after 1 month. After 1 year of drug discontinuation and treatment, his disease process remained, but without major progression. A Naranjo assessment score of 4 was obtained, indicating a possible relationship between the patient's adverse drug reaction and his use of the suspect drug.
\end{abstract}

Arjan Flora

Arjan.flora@utoledo.edu

1 Division of Pulmonary, Critical Care, and Sleep Medicine, Department of Internal Medicine, University of Toledo Medical Center, 3000 Arlington Ave, MS 1186, Toledo, OH 43614, USA

2 Pulmonary and Critical Care Specialists, The Toledo Clinic, Inc., 1661 Holland Rd, Maumee, OH 43537, USA

\section{Key Points}

There are no previous reports of interstitial pneumonitis due to desvenlafaxine despite being listed as an adverse drug reaction in the package insert.

Our case describes the attempted treatment for desvenlafaxine-induced interstitial pneumonitis and a comparison of known cases of venlafaxine-induced interstitial pneumonitis in the literature.

\section{Introduction}

Desvenlafaxine was introduced in 2008 as a once-a-day, single-dose, synthetic formulation of a major active metabolite of venlafaxine, a serotonin-norepinephrine reuptake inhibitor (SNRI). Venlafaxine-associated pulmonary toxicities are rare, and incidences are unknown despite widespread global use of the drug. There have only been a handful of cases of venlafaxine-induced interstitial pneumonitis since it became available on the market in 1993, with the condition currently being listed as a rare adverse effect in the drug safety literature. However, there is currently no established link between pulmonary toxicity and derivatives of the drug. We report a case of druginduced interstitial pneumonitis believed to be associated with desvenlafaxine. 


\section{Case Summary}

A 52-year-old Caucasian male with a past medical history significant for obstructive sleep apnea treated via continuous positive airway pressure (CPAP) and a remote history of right-sided viral pneumonia with adenopathy and hypoxemia 4-5 years prior presented with complaints of bronchitic symptoms and mild, persistent dyspnea over the past several months. He denied postnasal drip or gastroesophageal reflux disease (GERD)-related signs and symptoms. He had a distant smoking history of less than 10 pack-years, quitting 24 years prior. He worked a desk job at a glass-cutting factory, with exposures around 100 yards from his office consisting of silicate dust, wood dust from free-made crates, and heavy walnut flour used as packaging. Physical examination was only notable for very soft, low-pitched bibasilar wheezing with forced expiratory maneuver only. Pulmonary function testing found a newly developed restrictive pattern (Table 1). A chest X-ray showed diffuse bilateral small micronodular interstitial infiltrates without honeycombing; distinctly different from previous imaging years ago.

He was initially given a high-dose inhaled corticosteroid-long-acting beta-agonist combination inhaler for possible hypersensitivity bronchitis. A computed tomography (CT) chest scan showed a pattern of bilateral upper lobe mosaic attenuation and diffuse bibasilar predominant ground-glass and peripheral reticular opacities with faint traction bronchiectasis suggesting non-specific interstitial pneumonia or possible hypersensitivity pneumonitis (Fig. 1). Repeat spirometry was largely unchanged. Open lung biopsy of the right middle and lower lobes via videoassisted thoracoscopy revealed end-stage interstitial fibrosis with honeycomb changes, fibroblastic foci, and patchy chronic inflammatory infiltrate with lymphoid follicles presumed to be chronic hypersensitivity pneumonitis.

Re-review of the patient's environmental and occupational exposures and medication list (which included levothyroxine, omeprazole, glucosamine-chondroitin, fish oil, and a multivitamin with iron) found a temporal correlation between the onset of symptoms and the patient having his desvenlafaxine dose increased from $50 \mathrm{mg}$ orally (PO) daily to $100 \mathrm{mg} 6$ months prior to presentation. $\mathrm{He}$ had started on desvenlafaxine 4 years before. This medication was immediately discontinued 6 months after presentation, and prednisone was then started at $10 \mathrm{mg}$ daily with trimethoprim sulfamethoxazole prophylaxis, which aided in improvement of symptoms. Dyspnea was noted only when ambulating uphill and with brisk exercise. A mild cough remained, however, related to seasonal allergies. Bibasilar rales were noted on exam. Repeat pulmonary function testing a month after starting steroids showed minimal improvement (Table 1). Repeat highresolution CT chest continued to show bilateral upper lobe and basilar predominant disease with interstitial changes and some peripheral honeycomb effects.

Two months later, the patient was started on mycophenolate mofetil $1500 \mathrm{mg}$ PO twice daily (BID) with a slow taper of steroids of 1-year duration. During this time, the patient was evaluated at a tertiary university medical center with an interstitial lung disease clinic, where a connective tissue disease-related interstitial lung disease was ruled out, but they could not definitively rule in a drug-induced process. Despite discontinuation of the possible inciting medication and treatment with immunosuppressing agents without further changes in environmental or occupational exposures, the disease process was deemed already advanced by the time it was discovered. The patient felt that his breathing was slightly worse, but not debilitating.
Table 1 Pulmonary function trend

\begin{tabular}{lllll}
\hline PFTs & 3 years prior & On presentation & 1 month after Tx & 1 year after Tx \\
\hline FVC & $5.2(101 \%)$ & $3.53(69 \%)$ & $3.43(68 \%)$ & $3.14(62 \%)$ \\
FEV $_{1}$ & $4.35(108 \%)$ & $2.98(76 \%)$ & $3.1(80 \%)$ & $2.53(65 \%)$ \\
FEV $_{1} /$ FVC & $78 \%$ & $77 \%$ & $77 \%$ & $77 \%$ \\
FEF 25-75\% & $5(127 \%)$ & $4.3(127 \%)$ & $4.42(131 \%)$ & $2.36(71 \%)$ \\
SVC & $5.01(100 \%)$ & $3.38(67 \%)$ & $3.27(65 \%)$ & - \\
RV (Pleth) & - & $1.26(59 \%)$ & $1.19(56 \%)$ & - \\
TLC (Pleth) & - & $4.64(66 \%)$ & $4.46(63 \%)$ & - \\
DLCOunc & $68 \%$ & $36 \%$ & $49 \%$ & - \\
\hline
\end{tabular}

DLCOunc diffusion capacity of carbon monoxide, uncorrected (percent predicted), FEF 25-75\% forced expiratory flow between 25 and $75 \%$ of vital capacity [liters per second (percent predicted)], $F E V_{1}$ forced expiratory volume in $1 \mathrm{~s}$ [liters (percent predicted)], $F E V_{l} / F V C$ ratio of forced expiratory volume to forced vital capacity (\%), $F V C$ forced vital capacity [liters (percent predicted)], PFT pulmonary function test, $R V$ (Pleth) residual volume via plethysmography [liters (percent predicted)], SVC slow vital capacity [liters (percent predicted)], TLC (Pleth) total lung capacity via plethysmography [liters (percent predicted)], $T x$ treatment 

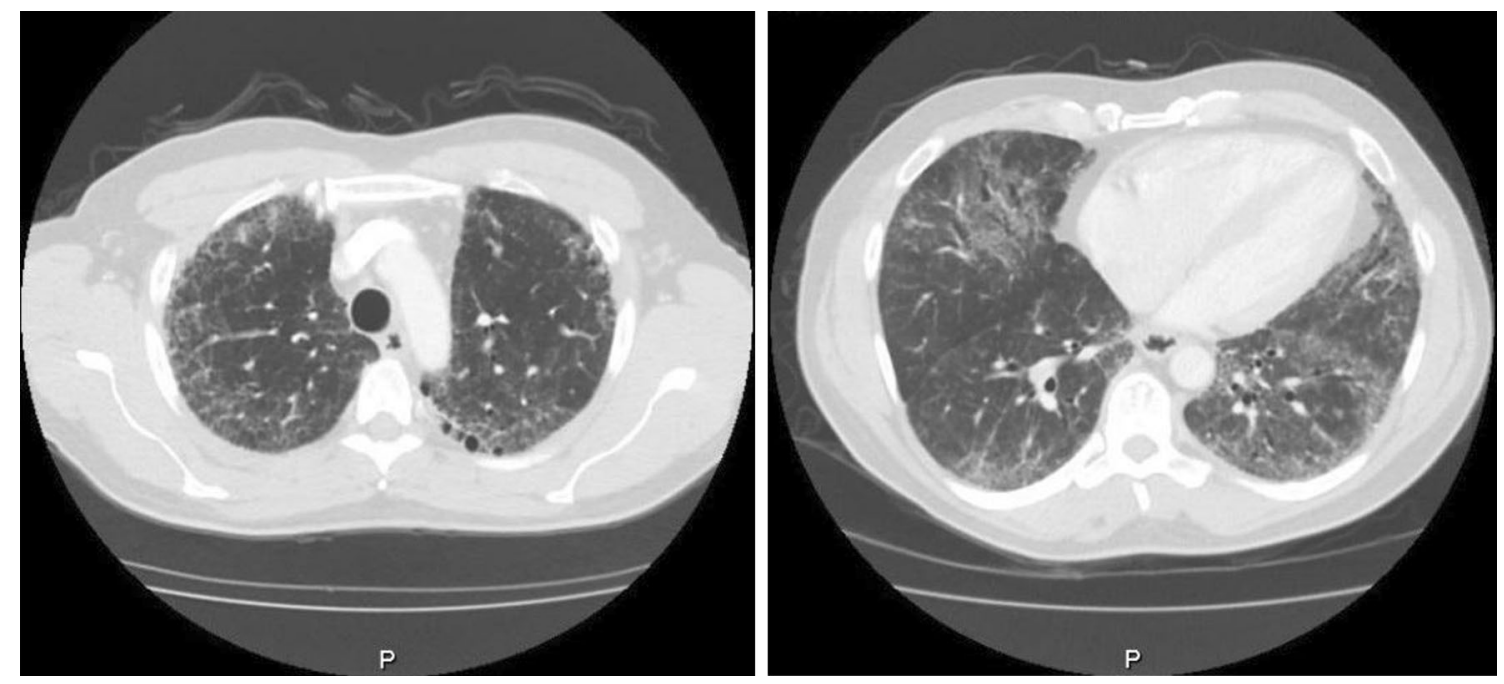

Fig. 1 CT chest scan at presentation. $C T$ computed tomography
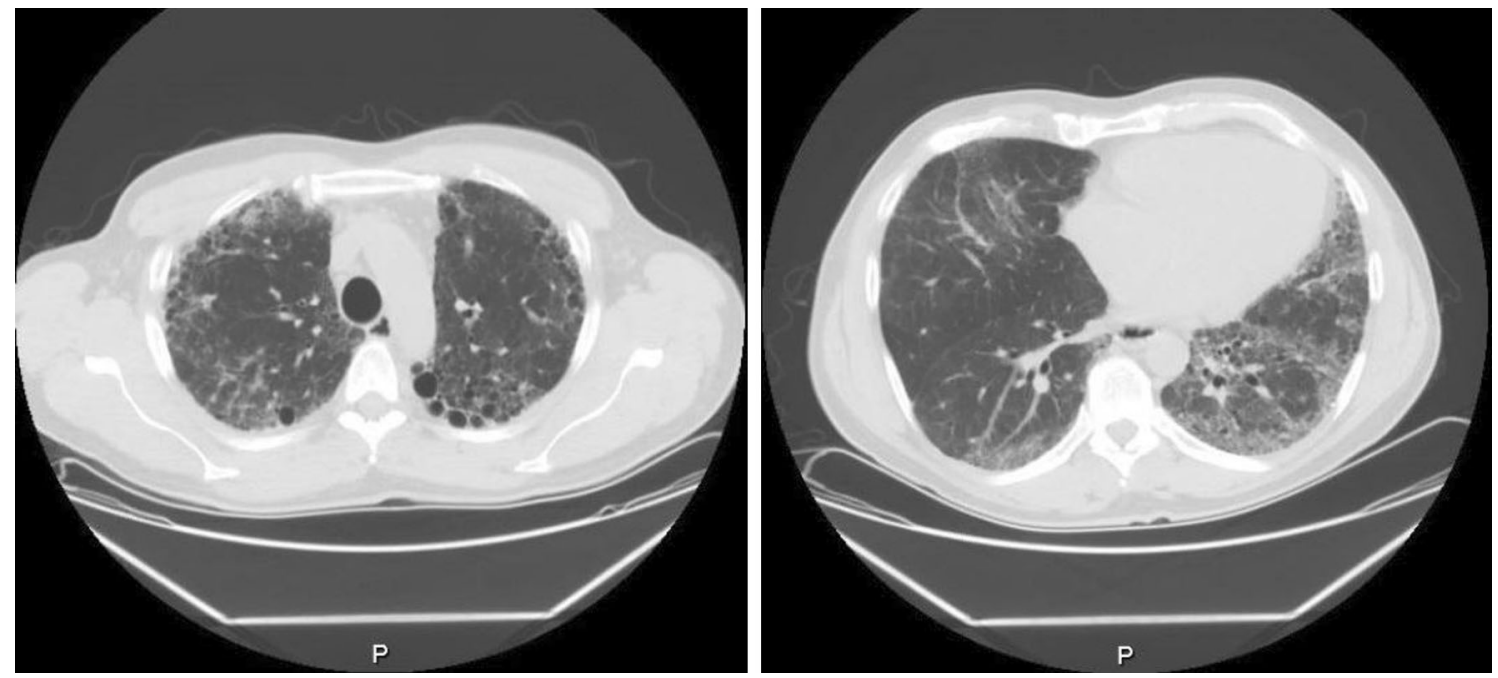

Fig. 2 CT chest scan 1 year after treatment with mycophenolate $1500 \mathrm{mg}$ PO BID and prednisone $10 \mathrm{mg}$ PO daily. BID twice daily, $C T$ computed tomography, $P O$ oral

Repeat pulmonary function testing and CT imaging (Fig. 2) demonstrated a slowing of the progression of disease, but notable subtle worsening. The patient is currently being considered as a potential candidate for anti-fibrotic therapy with nintedanib in the future.

\section{Discussion}

The SNRI venlafaxine has been used for major depressive disorder, anxiety, and panic disorder for over 2 decades. In that time, there have been at least seven cases of venlafaxine-induced interstitial pneumonitis reported in the medical literature (Table 2) [1-6], along with a smaller number of other venlafaxine-associated pulmonary toxicities such as eosinophilic pneumonia and even asthma. Pulmonary disease has become a well-established adverse effect of venlafaxine, and thus has been included in the prescribing information for its derivative, desvenlafaxine. This may be a class effect, as evident by reports of similar findings with selective serotonin reuptake inhibitors, such as fluoxetine [7].

The causal link between drug intake and an idiosyncratic reaction is usually difficult to recognize, especially in cases of patients treated with multiple medications. Several criteria must be fulfilled to establish that a drug causes disease: a temporal relation between drug commencement and disease onset, the clinical manifestations of disease characteristics, and clinical improvement after drug discontinuation. Using the Naranjo algorithm [8] to calculate an adverse drug 


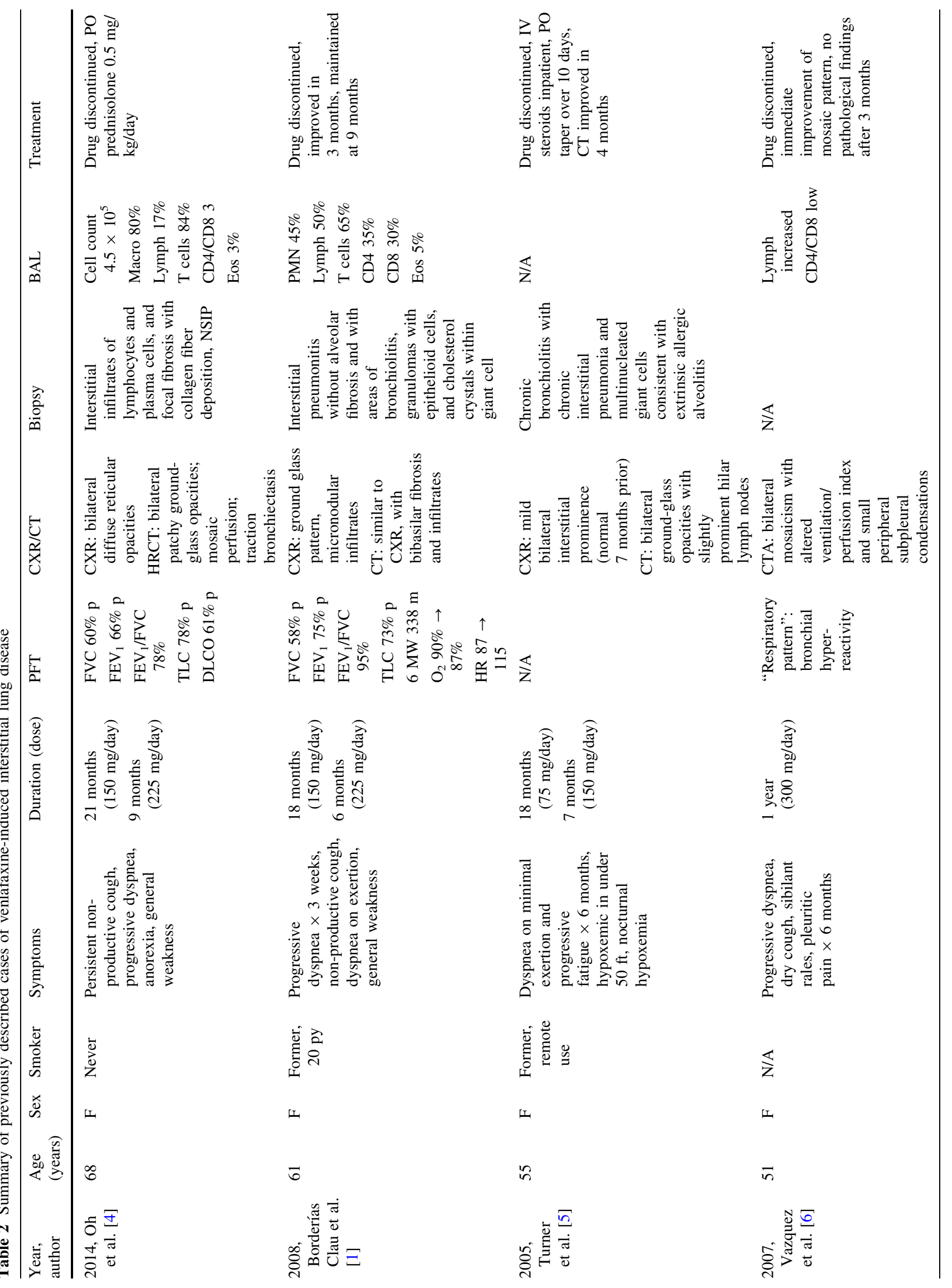




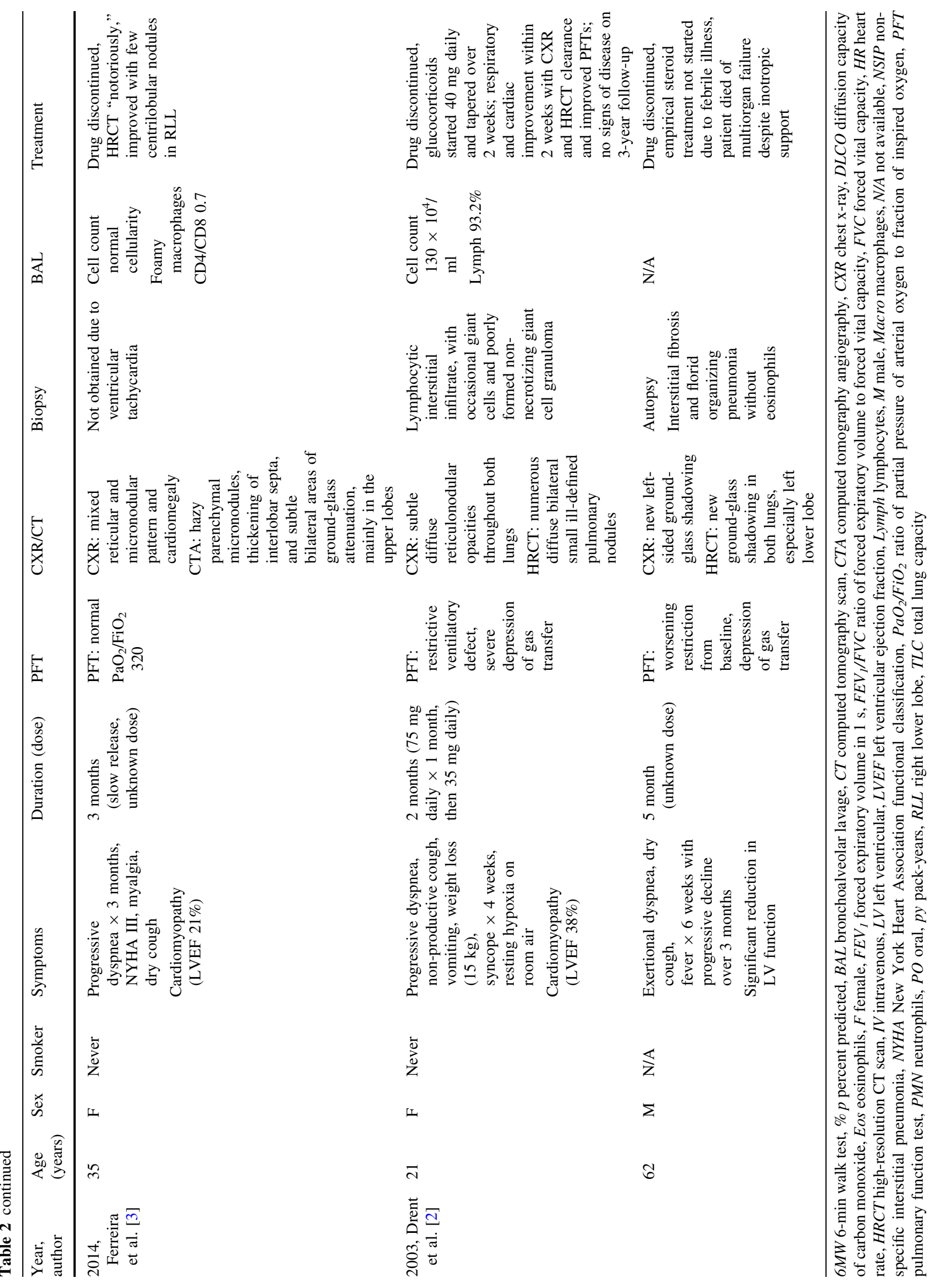


reaction probability score, our patient had a score of at least 4 , suggesting a "possible adverse drug reaction." We had no desire to challenge the patient by re-introducing the medication to him. The patient also did not want to be placed again on antidepressants for long-term major depressive disorder management, although anxiolytics were offered.

Hypersensitivity pneumonitis traditionally has been associated with inhalation of organic substances, industrial chemicals, and drugs. All previously reported cases of venlafaxine-induced interstitial pneumonitis exhibited radiological and pathological features consistent with hypersensitivity pneumonitis. Just as our patient demonstrated bilateral mosaic attenuation, ground-glass and reticular opacities, and traction bronchiectasis, six of the previously described cases featured similar radiographic findings. Open lung biopsy performed on our patient displayed lymphoid interstitial infiltrates such as in four of the above cases. While most of the previous cases presented as an acute or subacute interstitial pneumonitis, our patient's findings of focal fibrosis consistent with chronic pneumonitis were noted in only two other cases.

Interestingly, three patients were on lower doses of venlafaxine (75-150 mg daily) for 18-21 months, but were noted to only develop symptoms 6-9 months after a dose increase (150-225 mg daily). Those patients who developed cardiomyopathy did so within 3-5 months of starting their medication. A direct toxic effect and hypersensitivity reaction may be involved in venlafaxine-induced interstitial lung disease. Seventy percent of venlafaxine is metabolized in the liver by CYP2D6 into its metabolite $O$ desmethylvenlafaxine (desvenlafaxine), thus the effects of the two drugs are expected to be the same [9].

Desvenlafaxine is prescribed as a single dose of $50 \mathrm{mg}$ daily, and does not require incremental dosage increase for therapeutic effect as with venlafaxine. Interestingly, our patient only began to develop evidence of pulmonary disease after a doubling of the dose. As we continue to investigate our patient's disease, which demonstrated a relatively low Naranjo score, with a tertiary university center specializing in interstitial lung disease, we are yet to report the case to a national system of pharmacovigilance. It is important to report such findings for both new drugs as well as derivatives of older drugs for post-marketing drug monitoring. This can help create better insight into potential adverse effects that patients may develop while taking these medications.

\section{Conclusion}

We present a case of a middle-aged man who presented with signs and symptoms of interstitial pneumonitis 6 months after having a doubling of his dose of desvenlafaxine, which he had been on for 4 years. Despite drug discontinuation and treatment with immunosuppressive agents, the extent of his lung damage persisted while the progression itself slowed down. The drug is a synthetic derivative of one of the metabolites for venlafaxine, which has been previously reported to be related to the development of pulmonary disease. We continue to investigate and treat the patient's pulmonary disease to determine the role of desvenlafaxine, considering a low Naranjo score despite temporality of onset with dose increase.

Funding No sources of funding were used to assist in the preparation of this study.

\section{Compliance with Ethical Standards}

Conflict of interest Arjan Flora, MD has no conflicts of interest that are directly relevant to the content of this study. Daniel Pipoly, MD has no conflicts of interest that are directly relevant to the content of this study.

Informed consent Written informed consent was obtained from the patient for publication of this case report. A copy of the written consent may be requested for review from the corresponding author.

Open Access This article is distributed under the terms of the Creative Commons Attribution-NonCommercial 4.0 International License (http://creativecommons.org/licenses/by-nc/4.0/), which permits any noncommercial use, distribution, and reproduction in any medium, provided you give appropriate credit to the original author(s) and the source, provide a link to the Creative Commons license, and indicate if changes were made.

\section{References}

1. Borderías Clau L, Marigil Gómez MA, Val Adán P, Marcén Letosa M, Biescas López R, Garrapiz López FJ. Hypersensitivity pneumonitis due to venlafaxine. Arch Bronconeumol. 2008;44(10):571-3.

2. Drent M, Singh S, Gorgeis A, et al. Drug-induced pneumonitis and heart failure simultaneously associated with venlafaxine. Am J Respir Crit Care Med. 2003;167:958-61.

3. Ferreira PG, Costa S, Dias N, et al. Simultaneous interstitial pneumonitis and cardiomyopathy induced by venlafaxine. J Bras Pneumol. 2014;40(3):313-8.

4. Oh S, Cha SI, Kim H, et al. A case of venlafaxine-induced interstitial lung disease. Tuberc Respir Dis. 2014;77:81-4.

5. Turner RC, Nelson JE, Roberts BT, et al. Venlafaxine-associated interstitial pneumonitis. Pharmacotherapy. 2005;25(4):626-9.

6. Vazquez M, Quevedo B. Pneumonitis related to venlafaxine. Psychomatics. 2008;49(1):84-5.

7. Deidda A, Pisanu C, Micheletto L, Bocchetta A, Del Zompo M, Stochino ME. Interstitial lung disease induced by fluoxetine: systematic review of literature and analysis of VigiAccess, EudraVigilance and a national pharmacovigilance database. Pharmacol Res. 2017;120:294-301.

8. Naranjo CA, Busto U, Sellers EM, et al. A method for estimating the probability of adverse drug reactions. Clin Pharmacol Ther. 1981;30(2):239-45.

9. Lemke TL, Williams DA. Foye's principles of medicinal chemistry. Philadelphia: Lippincott Williams \& Wilkins; 2012. 